Rev. Bras. Saúde Prod. Anim., Salvador, v.13, n.1, p.01-12 jan/mar, 2012 http://www.rbspa.ufba.br ISSN 15199940

\title{
Perfil tecnológico de sistemas de produção de leite resfriado
}

\author{
Technological profile of cooled milk production systems
}

\begin{abstract}
FERNANDES, Sérgio Augusto de Albuquerque ${ }^{1 *}$; FALEIRO, Amanda dos Santos ${ }^{2}$; FERRÃO, Sibelli Passini Barbosa ${ }^{1}$; VIEIRA, Viviane Figueiredo ${ }^{3}$; SOUZA, Dayana Rodrigues $^{4}$; NUNES, Leonardo Ribeiro ${ }^{3}$; SANTOS, Neomara Brito de Lisboa ${ }^{5}$; FERRÃO, Iram da Silva ${ }^{6}$; PEREIRA, Marcelo Mota ${ }^{3}$; FREITAS, Mazzilli Amaral ${ }^{8}$; MATARAZZO, Soraia Vanessa ${ }^{7}$
\end{abstract}

\footnotetext{
${ }^{1}$ Universidade Estadual do Sudoeste da Bahia, Departamento de Tecnologia Rural e Animal, Itapetinga, Bahia, Brasil.

${ }^{2}$ Universidade Estadual do Sudoeste da Bahia, Programa de Pós-Graduação em Engenharia de Alimentos, Itapetinga, Bahia, Brasil.

${ }^{3}$ Universidade Estadual do Sudoeste da Bahia, Programa de Pós-Graduação em Zootecnia, Itapetinga, Bahia, Brasil.

${ }^{4}$ Secretária da Agricultura de Nova Ibiá, Bahia, Brasil.

${ }^{5}$ Universidade Federal do Recôncavo Baiano, Programa de Pós-Graduação em Zootecnia, Cruz das Almas, Bahia, Brasil.

${ }^{6}$ Agência Estadual de Defesa Agropecuária da Bahia, Itapetinga, Bahia, Brasil.

${ }^{7}$ Universidade Estadual de Santa Cruz, Departamento de Ciências Agrárias e Ambientais, Ilhéus, Bahia, Brasil.

${ }^{8}$ M. Sc. em Zootecnia - Bolsista FAPESB - DIT.

*Endereço para correspondência: sfernandes@uesb.edu.br
}

\section{RESUMO}

Objetivou-se com este trabalho caracterizar os fornecedores de leite de um laticínio comercial localizado no Território de Identidade de Itapetinga por meio da aplicação de um questionário semiestruturado. Do total de 28 fornecedores foram entrevistados 17, estes estratificados em pequenos (até $50 \mathrm{~kg}$ de leite/dia), médios (51 a 200kg de leite/dia) ou grandes (maior que $200 \mathrm{~kg}$ de leite/dia). A análise dos dados foi realizada por meio de análises unidimensionais e análises não paramétricas e comparação de médias. Não foram observados pequenos fornecedores de leite. Dos entrevistados, 17,6\% foram caracterizados como médios e $82,4 \%$ como grandes fornecedores de leite. O tempo médio na atividade leiteira variou entre 20 e 22 anos, respectivamente para grandes e médios fornecedores de leite. O agrupamento genético observado nos dois estratos foi o cruzamento Zebu X Taurinos, com produção média de $1.260 \mathrm{~kg} / \mathrm{leite} / \mathrm{lactação} \mathrm{e} 1.594 \mathrm{~kg} / \mathrm{leite} / \mathrm{lactação}$, respectivamente para os médios e grandes fornecedores. O período de lactação foi semelhante entre os estratos (274 dias). A idade ao primeiro parto variou entre 28 (médios) e 25 meses (grandes). O intervalo de partos foi de 406 dias (médios) e 480 dias (grandes). Todos os fornecedores entrevistados vacinavam seus animais contra febre aftosa. Apenas um fornecedor realiza o teste com tuberculina. Os sistemas de produção estudados apresentam deficiência no uso de tecnologias disponíveis e necessitam de planejamento para melhorar os indicadores zootécnicos.

Palavras-chave: diagnóstico, enfoque sistêmico, profilaxia, sanidade

\section{SUMMARY}

This study aimed to characterize the dairy producers in Territory of Itapetinga region using a semi-structured questionnaire. Seventeen dairy producers were interviewed and stratified into: small (up to $50 \mathrm{~kg}$ milk/day), medium (51 to $200 \mathrm{~kg}$ of milk/day) and large (more than $200 \mathrm{~kg}$ of 
milk/day). The data analysis was performed using one-dimensional analysis and nonparametric analysis. Small producers have not been identified. Of all dairy producers interviewed, $17.6 \%$ were characterized as medium and $82.4 \%$ as large dairy producers. The average time in dairy activity was 22 years to medium dairy producers and 20 years to large dairy producers. The herd genetic composition was crossbreed Zebu $\mathrm{x}$ Taurus. The average milk production was $1260 \mathrm{~kg} / \mathrm{milk} / \mathrm{lactation}$ to medium dairy producers and $1594 \mathrm{~kg} / \mathrm{milk} / \mathrm{lactation}$ to large dairy producers. The lactation period was 274 days to both strata. The age at first calving ranged from 25 to 28 months, respectively, to large or medium dairy producers. The calving interval was 406 days to medium dairy producers and 480 days to large producers. All interviewed dairy producers vaccine their herds against foot and mouth disease. Only one dairy producer realizes the tuberculin's test. The production systems evaluated showed low use of technologies and require a strategic management to improve animal production.

Keywords: healthiness, prophylaxis, ruminal diagnosis, system approach

\section{INTRODUÇÃO}

A produção mundial de leite em 2008 foi de aproximadamente 599 bilhões de litros, destes, o Brasil produziu mais de 30,7 bilhões, o que o coloca como o sexto maior produtor do mundo (CARVALHO \& CARNEIRO, 2012). No entanto, de acordo com esses autores, apesar de ser o sexto maior produtor de leite do mundo, o Brasil possui baixa produtividade, com apenas 1.3 ton. de leite por vaca/lactação.

Segundo Zoccal et. al. (2008), apesar do crescimento observado na produção de leite nos últimos anos, o consumo per capita brasileiro $(403 \mathrm{~mL} /$ habitante/dia) é inferior ao recomendado pelo Ministério da Saúde (663mL/habitante/dia), sendo um grande desafio a elevação da produção de leite para atender à demanda interna, estimada em 45 bilhões de litros de leite. Nesse sentido, duas vertentes devem ser levadas em consideração: a melhoria da qualidade do leite, em seus aspectos higiênico-sanitários; e aumento da quantidade em litros de leite produzido nos sistemas de produção, sem deixar de observar contudo, a interdependência entre esses dois aspectos que deve ser observada.

$\mathrm{O}$ enfoque atual na produção de leite relaciona-se ao novo paradigma, no qual o aumento na eficiência econômica da atividade pecuária, obtida por meio de aumento na produtividade dos fatores de produção, passa a ser buscada (STROPASOLAS, 2007).

A evolução do uso do enfoque sistêmico na agricultura é evidente. Este fato, de acordo com Pinheiro (2000), ocorre em função da complexidade dos sistemas organizados, o que tem requerido abordagem holística e multidisciplinar. Assim, a análise sistêmica instrumentaliza o pesquisador, orienta os agentes sociais e as políticas públicas no sentido da construção de novos saberes para fortalecer a multi e a interdisciplinaridade.

Baseado nesta premissa é importante a realização de diagnóstico dos sistemas de produção de leite resfriado de fornecedores de um laticínio comercial (entendido como um conjunto de partes inter-relacionadas que visa a atender um objetivo) do Território de Identidade de Itapetinga, Bahia.

\section{MATERIAL E MÉTODOS}

A metodologia utilizada para realização deste trabalho foi baseada na aplicação de questionário individual semiestruturado com produtores de leite. Foram entrevistados 17 produtores ligados a um laticínio comercial no Território de Identidade de Itapetinga (BA), no ano de 2007, que possuem tanques de 
resfriamento de leite por expansão direta, no total de 27. Os demais não responderam ao questionário por não se interessarem. Os temas abordados nas entrevistas foram relacionados aos atributos da propriedade, do produtor e de sua família. Para caracterizar a atividade leiteira foram considerados o rebanho, a produção leiteira, a sanidade, a reprodução, o manejo alimentar e mão de obra das propriedades. Também foi caracterizado o nível tecnológico, a diversificação produtiva e produção utilizada. Outros temas abordados na entrevista relacionaram-se às fontes de informações técnicas utilizadas pelos produtores, perspectivas futuras em relação à pecuária de leite $\mathrm{e}$ relação produtor/mercado.

Para a representação dos sistemas de produção foi utilizada a estratificação dos produtores conforme a produção diária de leite, conforme descrição a seguir: pequeno produtor - até $50 \mathrm{~kg}$ de leite/dia; médio produtor - 51 a $200 \mathrm{~kg}$ de leite/dia; grande produtor - acima de $200 \mathrm{~kg}$ de leite/dia.

As perdas na produção de leite em função do intervalo entre partos, por categoria de produtores, foram determinadas de acordo com a fórmula descrita por Ferreira et al. (2011):

$\mathrm{VPL}=(\mathrm{IPa}-\mathrm{IPd}) / \mathrm{IPd} X 100$, em que:

VPL = Variação na produção de leite $(\%)$;

$\mathrm{IPa}=$ Intervalo de Partos atual, e;

IPd = Intervalo de Partos desejado. Produção de leite por dia de intervalo de parto: produção de leite por lactação/número de dias de intervalos de parto.

Foi utilizado o método da tabela de contingência, seguida do teste Quiquadrado, para avaliar se o número de vacas em lactação e secas dependam do tipo de classificação das propriedades.
A produção de leite por lactação (litros), período de lactação, produção de leite por dia de intervalo entre partos e o período de serviço foram analisados por meio de testes não paramétricos, mediante o teste de Mann-Whitney para amostras independentes

A análise dos dados obtidos com o questionário foi realizada por meio de análises unidimensionais (OTANI et al., 1996), que consistem na tabulação em separado das respostas a cada pergunta, cujos resultados foram expressos em números absolutos (cabeças de animais por unidade de produção, por exemplo) ou percentuais (LUIZ \& SILVEIRA, 2000). Foi utilizado o pacote estatístico $\mathrm{R}$-DEVELOPMENT CORE TEAM (2010).

\section{RESULTADOS E DISCUSSÃO}

A região estudada compõe a segunda maior bacia leiteira da Bahia,com produção de mais de 230 milhões de litros de leite/ano (ZOCCAL, 2006; ADAB, 2008). Observou-se a inexistência de pequenos produtores, conforme classificação estabelecida, entre os sistemas de produção de leite estudados. Assim, das 17 propriedades cujos proprietários foram entrevistados, três $(17,6 \%)$ foram classificadas como médias e $14(82,4 \%)$ como grandes propriedades (Tabela 1). Provavelmente, o resultado encontrado neste trabalho se deva à posse da terra na região que é caracteristicamente latifundiária. De acordo com Manzano et al. (2006), mais de $50 \%$ do leite produzido no Brasil, origina-se de pequenos agricultores.

Em relação ao tamanho das propriedades, observou-se que as médias propriedades têm tamanho de 107ha; já as propriedades classificadas como grandes, possuem 
Rev. Bras. Saúde Prod. Anim., Salvador, v.13, n.1, p.01-12 jan/mar, 2012 http://www.rbspa.ufba.br ISSN 15199940

tamanho médio de 293ha. Em estudo similar na Bahia, Barreto et al. (2007) observaram tamanho médio de propriedades de 67,8ha e 216,5ha, para pequenos e médios produtores de leite, respectivamente. Neves et al. (2011) em estudos realizados nos sistemas de produção de leite no perímetro irrigado Senador Nilo Coelho no Sertão do São
Francisco, em Pernambuco, relatam que o tamanho médio das propriedades fornecedoras de leite observado foi de 14,4ha, com a área específica para a produção de leite de 8,9ha. Neste estudo, o tamanho médio variou entre 107 e 293ha (Tabela 1), respectivamente para médios e grandes produtores.

Tabela 1. Características dos fatores de produção dos sistemas de produção estudados

\begin{tabular}{|c|c|c|}
\hline \multirow{2}{*}{ Variáveis } & \multicolumn{2}{|c|}{ Categoria } \\
\hline & Médio (51-200 Kg/dia) & Grande (> $200 \mathrm{Kg} /$ dia) \\
\hline \multicolumn{3}{|l|}{ Fatores de produção } \\
\hline Número de propriedades & $3(17,6 \%)$ & $14(82,4 \%)$ \\
\hline Área média (ha) & 107 & 293 \\
\hline Tempo na atividade (anos) & $22(\mathrm{M} \mathrm{30} ; \mathrm{m} \mathrm{15})^{2}$ & $20(\mathrm{M} \mathrm{50} ; \mathrm{m} \mathrm{3})^{2}$ \\
\hline \multicolumn{3}{|l|}{ Empregos gerados (média) } \\
\hline Permanentes & $1,0(58,8 \%)$ & $2,9(55,7 \%)$ \\
\hline Temporários & $0,7(41,2 \%)$ & $2,3(44,3 \%)$ \\
\hline
\end{tabular}

${ }^{\mathrm{T}}$ Nenhum produtor se enquadrou na categoria com menos de $50 \mathrm{Kg} / \mathrm{dia} ;{ }^{2} \mathrm{M}$ : máximo; m: mínimo.

A diferença no tamanho das propriedades observado neste estudo e em relação aos resultados descritos por Neves et al. (2011), provavelmente é reflexo da região estudada que apresentam características de colonização diferentes da ocorrida no sertão pernambucano, em especial do perímetro irrigado, cujos lotes foram distribuídos por órgãos governamentais.

O tempo médio em que os produtores exploraram a atividade leiteira é de 22 anos e 20 anos para médios e grandes fornecedores, respectivamente. Os sistemas de produção classificados como grandes geram mais empregos que os médios, sejam os empregos permanentes (2,9 e 1,0, respectivamente), sejam temporários $(2,3$ e 0,7 , respectivamente). Pode-se inferir que o aumento na produção de leite determina aumento na necessidade de contratação de mão de obra. A região estudada caracteriza-se pelos baixos índices produtivos, em especial a produção de leite. Dessa forma, o aumento na produção de leite nos sistemas de produção, pode induzir a necessidade de contratação de mão de obra.

Em relação aos dados dos rebanhos e de acordo com os resultados observados (Tabela 2), o agrupamento genético predominante nos dois estratos foi $\mathrm{o}$ mestiço (Zebuínos X Taurinos). Neves et al. (2011) observaram presença maior de animais mestiços Holandês/zebu $(56,8 \%)$, Holandês $(20,4 \%)$, Gir $(15,9 \%)$ e sem padrão racial definido $(6,9 \%)$, ao estudarem sistemas de produção de leite na região do perímetro irrigado Nilo Coelho, em Pernambuco. A provável diferença existente entre os sistemas aqui relatados e os observados por Neves et al. (2011), talvez resida no maior uso de sistemas de produção intensivo, relatados por esses autores, diferente dos observados neste estudo, 
Rev. Bras. Saúde Prod. Anim., Salvador, v.13, n.1, p.01-12 jan/mar, 2012 http://www.rbspa.ufba.br ISSN 15199940

que caracterizam-se por sistemas extensivos.

A região aqui estudada caracteriza-se por pecuária extensiva, com predominância de sistemas de produção de gado de corte Neste contexto, os produtores entrevistados afirmam que utilizam animais azebuados em função da necessidade da venda dos machos, o que caracteriza um sistema misto de produção. Esta forma de produção induz a não especialização, que por sua vez determina baixos índices de produção e de uso de tecnologias disponíveis. No entanto, é observado na região um processo de mudança tecnológica.

Tabela 2. Características zootécnicas dos sistemas de produção de leite estudados

\begin{tabular}{lccc}
\hline \multirow{2}{*}{ Variáveis } & \multicolumn{3}{c}{ Categoria } \\
\cline { 2 - 4 } & $\begin{array}{c}\text { Média (51-200 } \\
\text { Kg/dia) }\end{array}$ & $\begin{array}{c}\text { Grande }(>200 \\
\text { Kg/dia) }\end{array}$ & $\begin{array}{c}\text { Valor de } \\
p^{1}\end{array}$ \\
\hline Grupamento genético & Mestiço & Mestiço & - \\
\hline Produção de leite & & & 1,000 \\
\hline Período de lactação, média (dias) & 274 & 274 & 0,232 \\
Kg/lactação & 1.260 & 1.594 & 0,285 \\
Kg/dia & 4,50 & 5,78 & 0,936 \\
Produção/leite/dia/IP & \\
Variação na produção de leite $(\%)$ & 3,01 & 3,33 & - \\
${ }^{\text {T}}$ Nível de significância de 5\%; ${ }^{2}$ Produção de leite por dia de intervalo de parto; ${ }^{3}$ Variação na produção de leite.
\end{tabular}

Os parâmetros produtivos de período de lactação, quilogramas de leite por lactação e por dia, produção de leite por dia de intervalos entre partos e a variação na produção de leite não diferiram entre os grupos estudados.

O período de lactação observado foi de 274 dias para os dois estratos. Verificou-se produção de $1.260 \mathrm{~kg}$ de leite/vaca/lactação entre os médios e $1.594 \mathrm{~kg}$ de leite/vaca/lactação entre os grandes produtores de leite, não foi observada diferença entre as médias ( $p>0,05)$. Estes resultados encontram-se acima dos relatos de Zoccal (2006) que afirma produção média na Bahia de $534 \mathrm{~kg}$ de leite/vaca/lactação. Os dados observados por Zoccal (2006) originamse do Instituto Brasileiro de Geografia e Estatística (IBGE), e refletem a produção de todo o rebanho, nesse caso, da Bahia. Por outro lado, as informações colhidas neste trabalho originam-se de produtores de leite, de uma das maiores regiões produtoras no Estado. Assim, provavelmente, esta diferença se dê em função do estrato de produtores avaliados.

O período de lactação, assim como a produção de leite entre os grupos determinam produções diárias de $4,5 \mathrm{~kg}$ (médios produtores) e $5,78 \mathrm{~kg}$ (grandes produtores), não foi observada diferença entre estes. A produção dos dois grupos é inferior ao observado por Neves et al. (2011), que relatam produção média de 9,3kg de leite/dia.

Barreto et al. (2007) ao estudarem sistemas de produção de leite na Bahia observaram produção diária com variação entre 1,68 e $2,02 \mathrm{~kg}$ de leite. Os valores relatados por esses autores, assim como os observados neste trabalho foram inferiores ao publicado por Manzano et al. (2006), que reportaram produções diárias de leite 
em sistemas de produção familiar de 8 $\mathrm{Kg} / \mathrm{vaca} /$ dia a $18 \mathrm{~kg} / \mathrm{vaca} /$ dia.

Ao se cruzar os dados de produção de leite por lactação com o intervalo de partos, chega-se à produção real por animal, assim, têm-se produções de leite por dia de intervalo de partos (produção de leite por lactação/intervalo de partos) de 3,01 e $3,33 \mathrm{~kg}$ de leite para médios e grandes produtores, respectivamente. Este parâmetro é importante para se visualizar a real produtividade. Verificase diminuição na produtividade em ambos os grupos. No grupo de médios produtores é observada diminuição de $31,1 \%$ de leite, quando se compara a produção dia com a produção por dia de intervalo entre partos. Por sua vez, no grupo de grandes produtores foi observada redução de $42,4 \%$, ao se elaborar este índice. Este índice é importante para que os envolvidos com a produção de leite da região estudada verifiquem onde estão os erros e busquem resolvê-los.

De posse dos resultados obtidos nestes parâmetros, é possível a determinação da variação na produção de leite. Verifica-se que no grupo de grandes produtores a variação média foi de $32 \%$. Entretanto existem fazendas do grupo de grandes produtores nas quais a variação chegou a $100 \%$ e outras em que a variação foi nula. Por outro lado, no grupo de médios produtores, a variação média foi de $17 \%$, e chegou também a ser nula em um dos sistemas de produção. Este índice é influenciado diretamente pelo intervalo de partos, assim, se o intervalo de partos é de 24 meses (um dos casos), sua diminuição para o intervalo ideal (12 meses) proporcionaria elevação de $100 \%$ na produção de leite.

De acordo com Silva et al. (2006) existem características reprodutivas que são afetadas pela vaca (primeira cobertura em novilhas, ou intervalo parto - primeiro cio), características que são afetadas por reprodutores acasalados com vacas (fertilidade do macho) e características que são afetadas por ambos (taxa de não retorno ao cio, número de inseminações por concepção). No entanto, a expressão dessas características é influenciada pelo meio. Assim, mesmo que as características anteriormente citadas sejam satisfeitas, e as condições do meio não, a expressão gênica não se dá em sua plenitude. As condições de produção (meio) observadas nos sistemas avaliados, em sua maioria apresentam deficiências, que associadas às características genéticas dos animais refletem-se nos baixos índices aqui relatados.

O percentual de vacas lactantes e secas pode ser observado na Figura 1. Não foi verificada dependência entre $o$ número de vacas lactantes ou não e a classificação das propriedades. Nas propriedades médias o percentual de vacas em lactação é de $67,17 \%$ e as secas $32,83 \%$, por sua vez, nas propriedades grandes, estes percentuais são $65,28 \%$ e $34,72 \%$, para lactantes e secas, respectivamente. De acordo com Neves et al. (2011) o percentual ideal de vacas em lactação é de $83 \%$. Assim, em ambos os estratos o percentual de vacas lactantes encontra-se abaixo da recomendação técnica considerada ideal. Estes resultados demonstram a ineficiência reprodutiva dos rebanhos estudados, pois o percentual de vacas em lactação se relaciona diretamente com o intervalo de partos. Assim, se o intervalo entre partos é longo, o número de vacas paridas diminui, consequentemente, a produção leiteria também cai. Como o produto principal desses sistemas de produção é o leite, tem-se baixa produção por unidade produtiva (Tabela 2 ).

Manzano et al. (2006) realizaram estudos em sistemas de produção e 
Rev. Bras. Saúde Prod. Anim., Salvador, v.13, n.1, p.01-12 jan/mar, 2012 http://www.rbspa.ufba.br ISSN 15199940

relataram percentual de vacas em lactação entre $44,4 \%$ e $81,5 \%$. Em apenas um dos sistemas estudados por esses autores, o percentual de vacas em lactação foi menor que os resultados observados neste trabalho. Neves et al. (2011), relatam percentual de vacas em lactação de 52,15\%, menor que o observado neste trabalho.

A complexidade do fenômeno reprodutivo é evidente por seus reflexos econômicos nos sistemas de produção animal. Dentre os parâmetros reprodutivos, não foi observada diferença estatística $(p<0,05)$ entre os grupos estudados (Tabela 3 ).

Entre as características reprodutivas, uma das mais importantes é a idade ao primeiro parto (RAMOS et al., 2004), que deve se situar entre 24 até 34 meses de idade (CAMPOS et al., 2006).

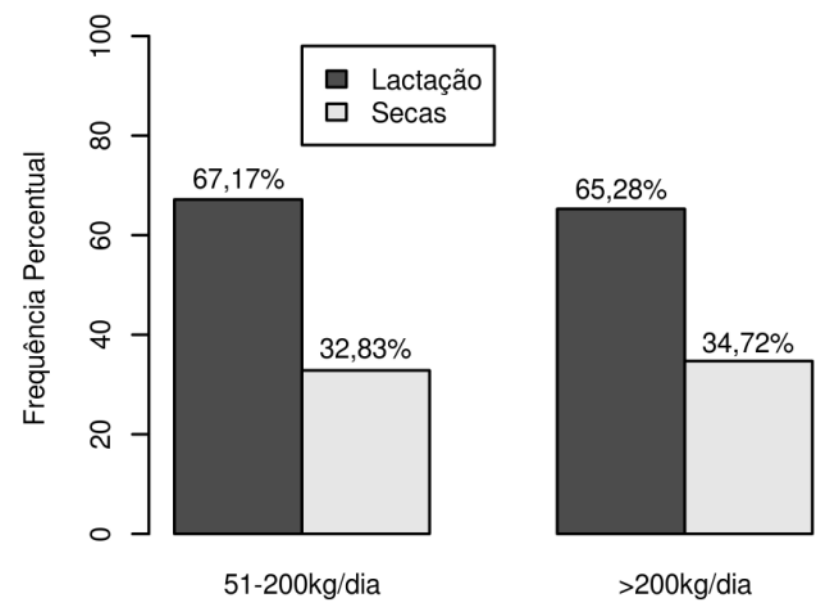

Figura 1. Frequência de vacas em lactação e secas, de acordo com a classificação estabelecida

Tabela 3. Indicadores reprodutivos observados nos sistemas de produção estudados

\begin{tabular}{lccc}
\hline \multirow{2}{*}{ Variáveis } & \multicolumn{3}{c}{ Categoria } \\
\cline { 2 - 4 } & $\begin{array}{c}\text { Média } \\
(51-200 \mathrm{Kg} / \text { dia })\end{array}$ & $\begin{array}{c}\text { Grande } \\
(>200 \mathrm{Kg} / \text { dia })\end{array}$ & Valor de $p^{1}$ \\
\hline Idade $1^{\circ}$ parto (meses) & 28 & 25 & 0,364 \\
Intervalo de partos (dias) & 406 & 480 & 0,062 \\
Período de serviço (dias) & 105 & 90 & 0,705 \\
Período de descanso (dias) & 75 & 72 & 0,879 \\
\hline
\end{tabular}

${ }^{\mathrm{T}}$ Foram utilizados os testes de Mann-Whitney e teste t de Student, com nível de significância de 5\%.

A idade ao primeiro parto observada neste trabalho, nas médias propriedades, foi de 28 meses e para as grandes, 25 meses, esta menor que a observada por Wolff et al. (2004), que ao estudarem os parâmetros reprodutivos em bovinos leiteiros (Holandês) no Paraná, relataram idade ao primeiro parto de 27 meses. Também foi menor que a observada por Marques et al. (2002) de 37 meses e por Facó et al. (2005) 33,8 meses. 
A idade ao primeiro parto nas médias e grandes propriedades encontra-se dentro do intervalo ideal (24 e 34 meses) considerado por Campos et al. (2006). Destaca-se que as características de fertilidade têm baixa herdabilidade, assim, tais características são mais influenciadas pelo ambiente (TONHATI et al., 2000).

Os indicadores reprodutivos se relacionam estreitamente com a eficiência econômica dos sistemas de produção (SILVA et al., 2006). Dentre estes, o intervalo entre partos é o de maior expressão, em função de $\mathrm{o}$ mesmo se relacionar com os demais parâmetros reprodutivos, como: taxa de concepção, período de serviço, prenhez, taxa de aborto, entre outros. O intervalo de partos em rebanhos leiteiros ideal é de 12 meses, ou 365 dias, e quanto mais longo, menor será e eficiência reprodutiva, traduzidas em perdas na eficiência econômica (VILELA et al., 2007). Ainda de acordo com esses autores, o intervalo de partos é reflexo direto do período de serviço (período entre o parto e a concepção, expresso em dias e, que não deve ser maior que 90 dias), e a gestação. Esta condição poderá ser obtida com eficiente programa sanitário preventivo, associado à alimentação adequada.

O intervalo de partos observado nas médias e grandes propriedades foi de 406 dias e 480 dias, respectivamente. Facó et al. (2005) ao estudarem o intervalo de partos em diferentes grupos genéticos de bovinos, observaram intervalos de partos que variaram entre 409 e 433 dias. Esses autores ainda relatam que, em rebanhos mestiços (não especializados) é importante que o intervalo entre partos seja diminuído, visto que a extensão da lactação é menor que em rebanhos especializados, como os de raça Holandesa.
O período de serviço (PS) - intervalo entre $\mathrm{o}$ parto e a primeira cobertura fértil - observado foi de 105 e 90 dias, nas médias e grandes propriedades, respectivamente, indicado que se mantenha entre 60 e 90 dias (AZEVEDO et al., 2006). Existe relação direta entre o período de serviço e o intervalo de partos, e uma forma de se diminuir o intervalo de partos é encurtar o PS (VILELA et al., 2007). À medida que o PS se encontra acima de 90 dias o intervalo de partos se dilata, e quando o IP se encontra acima de 12 meses, as perdas de leite evidenciam-se. Estas podem ser avaliadas por meio da variação na produção de leite (Tabela).

As perdas na produção de leite observadas neste trabalho por meio da variação na produção de leite (VPL, \%) para cada mês a menos no intervalo de partos, foram descritas na Tabela 2. Entre os médios produtores a variação na produção de leite foi de $1,3 \%$, e entre os grandes produtores 30,95\%. Dessa forma, as perdas na produção de leite, em função do intervalo de partos, entre os grandes fornecedores é grande, traduzindo-se em grande ineficiência econômica.

As variáveis sanitárias são importantes na produção animal, visto que os produtos de origem animal como carne e leite, podem ser fontes de infecção de micro-organismos com caráter zoonótico, além de estarem ligados à eficiência econômica dos sistemas de produção.

As medidas profiláticas adotadas pelos produtores podem ser analisadas ao se observar a Tabela 4.

A febre aftosa (Aphtovirus) é uma doença animal que tem sido fonte de preocupação por parte das autoridades sanitárias do país. Assim, ocorre anualmente campanhas de vacinação obrigatórias em todo o território nacional. No Nordeste do Brasil, apenas a Bahia e Sergipe possuem "status" de livre de febre aftosa com 
Rev. Bras. Saúde Prod. Anim., Salvador, v.13, n.1, p.01-12 jan/mar, 2012 http://www.rbspa.ufba.br ISSN 15199940

vacinação. De acordo com as respostas obtidas, os produtores têm acompanhado as vacinações oficiais, visto que $100 \%$ dos mesmos vacinam seus animais (Tabela 4). Neves et al. (2011), relatam que $96,4 \%$ dos produtores entrevistados na região de Petrolina (PE) vacinam seus animais contra a febre aftosa, percentual similar ao observado neste estudo. Porém, Fernandes et al. (2008), quando estudaram sistemas de produção de bubalinos na Zona da Mata de Pernambuco, relataram que apenas $54 \%$ dos produtores vacinavam os animais contra febre aftosa.

Tabela 4. Práticas profiláticas observadas nos sistemas de produção de leite estudados

\begin{tabular}{lcc}
\hline \multirow{2}{*}{ Variáveis } & \multicolumn{2}{c}{ Categoria } \\
\cline { 2 - 3 } & Média $(51-200 \mathrm{Kg} /$ dia $)$ & Grande $(>200 \mathrm{Kg} /$ dia $)$ \\
\hline Práticas profiláticas & $3(100,0 \%)$ & $14(100,0 \%)$ \\
\hline Febre aftosa & $3(100,0 \%)$ & $13(92,8 \%)$ \\
Brucelose & $3(100,0 \%)$ & $12(85,7 \%)$ \\
Raiva & $3(100,0 \%)$ & $12(85,7 \%)$ \\
Carbúnculo sintomático & $0(0,0 \%)$ & $1(7,1 \%)$ \\
Tuberculinização & & \\
\hline
\end{tabular}

Na mesma tabela, observa-se que todos os produtores classificados como médios praticam a vacinação contra brucelose (Brucella abortus) de acordo com as recomendações do Ministério da Agricultura, Pecuária e Abastecimento (2006). Entre os grandes produtores 92,8\% imunizam seu rebanho de acordo com a legislação e apenas um não o faz, o que demonstra, dessa forma, a preocupação dos produtores em relação a esta zoonose, uma vez que segundo Silva et al. (2007), $45 \%$ dos casos humanos de brucelose reportados na Califórnia (EUA) entre 1973 e 1992, foram oriundos do consumo de produtos lácteos contaminados. Neves et al. (2011), na região de Petrolina (PE) observaram que apenas $17,4 \%$ dos produtores de leite estudados vacinavam seus animais contra brucelose. Fernandes et al. (2008) relatam que apenas $41,7 \%$ dos produtores de búfalos vacinam seus animais contra brucelose.
Em relação à tuberculose bovina (Mycobacterium bovis), apenas um produtor entre todos os entrevistados realiza o teste com tuberculina. De acordo com Silva et al. (2007) a infecção pelo Mycobacterium bovis é responsável por aproximadamente 5\% dos casos humanos de tuberculose no Brasil, isto evidencia a importância do controle desta doença. Com relação às doenças cuja vacinação não é obrigatória como Raiva e Carbúnculo Sintomático, os médios produtores vacinam $100 \%$ de seu rebanho, já nas propriedades classificadas como grandes $85,7 \%$ dos fornecedores vacinaram seus rebanhos.

Diante destas informações torna-se necessário que os profissionais da extensão atuem como mediadores e facilitadores de processos de mobilização e organização de diferentes grupos de interesses (produtores de leite), e não propriamente os agentes propulsores/condutores desses processos (STROPASOLAS, 2007). 
Rev. Bras. Saúde Prod. Anim., Salvador, v.13, n.1, p.01-12 jan/mar, 2012 http://www.rbspa.ufba.br ISSN 15199940

Para Dutra \& Erdman (2007) uma das principais ferramentas para se atingir a eficiência econômica é o planejamento e controle da produção. Deficiências projetam-se de forma imediata na qualidade do bem, ou serviço produzido, assim como em menor aproveitamento dos recursos de produção, enfim, na perda da eficiência e eficácia, traduzidas nos índices zootécnicos insuficientes observados nos sistemas de produção estudados.

Dessa forma, a sustentabilidade de um sistema de produção perpassa pela aquisição/desenvolvimento tecnológico, que busque manter a atividade produtiva, seja na agricultura familiar seja na empresarial. Assim, a produção tecnológica deve ser orientada sistemicamente, para que todo $\mathrm{o}$ segmento econômico se beneficie dos ganhos tecnológicos (ALEIXO et al., 2007).

Os sistemas de produção estudados apresentam deficiências no uso de tecnologias disponíveis e necessitam de planejamento para melhorar os indicadores zootécnicos.

\section{REFERÊNCIAS}

AGÊNCIA ESTADUAL DE DEFESA AGROPECUÁRIA DA BAHIA ADAB. Relatório Técnico: produção de leite inspecionado na Bahia, 2008. 28p.

ALEIXO, S.S.; SOUZA, J.G.; FERRAUDO, A.S. Técnicas de análise multivariada na determinação de grupos homogêneos de produtores de leite. Revista Brasileira

Zootecnia, v.36, n.6, p.2168-2175, 2007. Ssupl.
AZEVÊDO, D.M.M.R.; MARTINS

FILHO, R.; LÔBO, R.N.B;

MALHADO, C.H.M; LÔBO, R.B.;

MOURA, A.A.A.; PIMENTA FILHO,

E.C. Desempenho reprodutivo de vacas Nelore no Norte e Nordeste do Brasil. Revista Brasileira de

Zootecnia, v.35, n.3, p.988-996, 2006. Supl.

BARRETO, D.; NUNES, L.R.; FERNANDES, S.A.A.; PEDREIRA, M.S.; MUNIZ, L.M.S.; ALMEIDA, L.N.; DUARTE, R.A.B.; FREITAS, M.A.; MATARAZZO,

S.V.Características estruturais dos sistemas de produção de leite dos produtores ligados à Associação de Produtores de Leite de Itagiba, Bahia. Revista do Instituto de Laticínios “Cândido Tostes", v.62, n.357, p.451-458, 2007.

BRASIL. Ministério da Agricultura, Pecuária e Abastecimento. Programa Nacional de Controle e Erradicação da Brucelose e da Tuberculose Animal (PNCEBT) / Brasília : MAPA/SDA/DSA, 2006. 188p.

CAMPOS, A.L.T.; FERREIRA, A.M. Composição no rebanho e sua importância no manejo. 2. ed. revisada e atualizada. Instrução técnica para o produtor de leite. Embrapa, 2006. Disponível em: <http://www. cnpgl.embrapa.br/pastprod/32Instruca o.pdf>. Acesso em: 18 jul. 2010.

CARVALHO, G.R.; CARNEIRO, A.V. Principais indicadores leite e derivados. Juiz de Fora: Embrapa Gado de Leite, 2010. Disponível em: < http://www.infoteca.cnptia.embrapa.br /handle/doc/882231>. Acesso em: 10 mar. 2011. 
Rev. Bras. Saúde Prod. Anim., Salvador, v.13, n.1, p.01-12 jan/mar, 2012 http://www.rbspa.ufba.br ISSN 15199940

DUTRA, F.A.F.; ERDMANN, R.H. Análise do planejamento e controle da produção sob a ótica da Teoria da Complexidade. Produção, v.17, n.2, p.407-419, 2007.

FACO, O.; LOBO, R.N.B; MARTINS FILHO, R.; LIMA, F.A.M. Idade ao primeiro parto e intervalo de partos de cinco grupos genéticos Holandês x Gir no Brasil. Revista Brasileira Zootecnia, v.34, n.6, p.1920-1926, 2005.

FERNANDES, S.A.A.; LOPES, G.M.B.; PANIZZA, J.C.J.; MATARAZZO, S.V. Sistemas produtivos de búfalos na zona canavieira de Pernambuco, caracterização e diagnóstico. Revista Brasileira de Saúde e Produção Animal [Online], v.9, n.3, p.565-577, 2008.

FERREIRA, A.M.; SÁ, W.S.; VIANA, J.H.M.; CAMARGO, L.S.A.

Importância Econômica de intervalos entre partos. Disponível em: <http://www.agencia.cnptia.embrapa.br/ Agencia8/AG01/arvore/AG01_79_21720 039240.html>. Acesso em: 25 nov. 2012.

LUIZ, A. J.B.; SILVEIRA, M.A. Diagnóstico rápido e dialogado em estudos de desenvolvimento rural sustentável. Pesquisa Agropecuária Brasileira, v.35, n.1, p.83-91, 2000.

MANZANO, A.; NOVAES, N.J.; CAMARGO, A.C.; ESTEVES, S.N.; FREITAS, A.R. Efeitos da implantação de técnicas agropecuárias na intensificação de sistemas de produção de leite em estabelecimentos familiares.

Revista Brasileira de Zootecnia, v.35, n.2, p.618-628, 2006

MARQUES, V.M.; REIS, R.P.; SÁFADI, T.; REIS, A.J. Custo e escala na pecuária leiteira: estudos de casos em Minas Gerais. Ciência e Agrotecnologia, v.26, n.5, p.1027-1034, 2002.
NEVES, A.L.A.; PEREIRA, L.G.R.; SANTOS, R.D.; ARAÚJO, G.G.L.; CARNEIRO, A.V.; MORAES, S.A; SPANIOL, C.M.O.; ARAGÃO, A.S.L. Caracterização dos produtores e dos sistemas de produção de leite no perímetro irrigado de Petrolina/PE. Revista Brasileira de Saúde e Produção Animal [Online], v.12, n.1, p.209-223, 2011

OTANI, M.N; CARRIERI, A.P.; ANGELO, J.A. Microbacia-piloto da córrego de São Joaquim, DIRA de Campinas, Estado de São Paulo: um estudo comparativo 1988-94.

Informações Econômicas, v.26, n.1, p.47-60, 1996.

PINHEIRO, S.L.G. O enfoque sistêmico e o desenvolvimento rural sustentável: uma oportunidade de mudança da abordagem hard-systems para experiências som soft-systems.

Agroecologia e Desenvolvimento Rural Sustentável, v.1, n. 2, p.27-37, 2000.

R DEVELOPMENT CORE TEAM (2010). R: a Language and Environment for Statistical Computing. Vienna: R Foundation for Statistical Computing, 2010. Disponível em: <http://www.Rproject.org>. Acesso em: 10 nov. 2010.

RAMOS, A.A. WECHSLER, F.S.; ONSELEN, V.J.; GONÇALVES, H.C. Sumário de touros bubalinos. Botucatu: UNESP/FMVZ, 2004. 37p. (PROMEBUL Boletim Técnico, 2).

SILVA, M.R.; PORTES, V.M.; MENIN, A.; ALVES, F.S.F. Doenças transmitidas pelo leite e sua importância em saúde pública. Revista do Instituto de Laticínios "Cândido Tostes", v. 62, n.358, p. 3-18, 2007. 
Rev. Bras. Saúde Prod. Anim., Salvador, v.13, n.1, p.01-12 jan/mar, 2012 http://www.rbspa.ufba.br ISSN 15199940

SILVA, M.V.G.B.; COBUCI, J.A.; FERREIRA, W.J.; OLIVEIRA, P.R.P.; MACHADO, M.A.; FERREIRA, C.P.. Efeitos genéticos e de ambiente em um rebanho do ecótipo mantiqueira. I. Características reprodutivas. Ciência Agrotécnica, v.30, n.3, p.522-528, 2006.

STROPASOLAS, V.L. Desenvolvimento rural e inclusão social: os alcances da ação extensionista no projeto microbacias 2. Raízes, v.26, n.1-2, p.120-127, 2007.

TONHATI, H.; VASCONCELLOS, F.B.; ALBUQUERQUE, L.G. Genetics aspects of productive and reproductive traits in a Murrah buffalo herd in São Paulo, Brazil. Journal Animal Breeding Genetic, v.117, p.331- 336, 2000.

VILELA, D, FERREIRA, A.M;

RESENDE, J.C.; LIMA, J.A.; VERNEQUE, R.S. Efeito do concentrado no desempenho produtivo, reprodutivo e econômico de vacas da raça Holandesa em pastagem de coastcross. Arquivo Brasileiro de Medicina Veterinária e Zootecnia, v.59, n.2, p.443-450, 2007.
WOLFF, M.C.C.; MONARDES, H.G.; RIBAS, N.P. Fatores ambientais sobre a idade ao primeiro parto, dias abertos e intervalo entre partos em vacas da raça Holandesa na bacia leiteira de Castrolanda, estado do Paraná. Archives of Veterinary Science, v.9, n.2, p.35-41, 2004.

ZOCCAL, R. Produção, industrialização e comercialização de leite no Brasil. 2006. Disponível em: <http://www.cnpgl.embrapa.br/produca o/producao.php>. Acesso em: 03 maio 2007).

ZOCCAL, R; CARNEIRO, A.V.; JUNQUEIRA, R.; ZAMAGNO, M.A nova pecuária leiteira brasileira. In: CONGRESSO BRASILEIRO DE QUALIDADE DO LEITE, 3.. Recife, 2008. Anais... Recife: 2008. p.85-95.

Data de recebimento: 08/05/2011

Data de provação: 09/12/2011 